\title{
Global urban carbon networks: linking inventory to modelling
}

\author{
Shaoqing Chen ${ }^{1,2 *}$, Huihui Long ${ }^{1,2}$, Brian D. Fath ${ }^{3,4,5} \&$ Bin Chen $^{6 *}$
}

${ }^{1}$ School of Environmental Science and Engineering, Sun Yat-Sen University Guangzhou 510275, China

${ }^{2}$ Guangdong Provincial Key Laboratory of Environmental Pollution Control and Remediation Technology (Sun Yat-sen University), Guangzhou 510275, China

${ }^{3}$ Department of Biological Sciences, Towson University, Towson, MD 21252, USA

${ }^{4}$ Advanced Systems Analysis Program, International Institute for Applied Systems Analysis, Laxenburg, Austria

${ }^{5}$ Environmetnal Studies, Masaryk University, Brno, Czech Republic

${ }^{6}$ State Key Joint Laboratory of Environment Simulation and Pollution Control, School of Environment, Beijing Normal University, Beijing 100875, China

Corresponding authors:

chenshaoqing@mail.sysu.edu.cn (S. Chen)

chenb@bnu.edu.cn (B. Chen) 


\section{Abstract}

Cities utilize and manipulate an immense amount of global carbon flows through their economic and technical activities. Here, we establish the carbon networks of eight global cities

4 by tracking the carbon exchanges between various natural and economic components. The

5 metabolic properties of these carbon networks are compared by combining flow-based and

6 interpretative network metrics. We further assess the relations of these carbon metabolic

7 properties of cities with their socioeconomic attributes that are deemed important in urban

8 development and planning. We find that though there is a large difference in city-level carbon

9 balance and flow pattern, a similarity in inter-component relationships and metabolic

10 characteristics can be found. Cities with lower per capita carbon emissions tend to have

11 healthier metabolic systems with better cooperation amongst various industries, which indicates

12 there may be synergy between urban decarbonization and metabolic system optimization.

13 Combination of indicators from flow balance and network models is a promising scheme for

14 linking carbon inventories to metabolic modelling efforts. With this done, we may be able to

15 fill the knowledge gap in current practices of carbon mitigation priorities as to how various

16 carbon flows in cities can be concertedly managed according to urban economic and

17 demographic changes.

18 


\section{Introduction}

Cities are a major contributor to greenhouse gas emissions and will probably remain so in the foreseeable future. With an occupation of less than $3 \%$ of global land surface, ${ }^{1,2}$ urban areas account for approximately $70 \%$ of global carbon emissions owing to the concentration of production and consumption activities. ${ }^{3}$ In the coming decades, major growth of carbon emissions will take place in many cities around the world as long as these cities continue their carbon-intensive economic growth and land-use expansion, which is particularly the case for cities in less developed countries and regions. ${ }^{4,5}$ This poses a great challenge with regard to achieving the $1.5^{\circ} \mathrm{C}$ global climate target ${ }^{6}$ and the United Nations Sustainable Development Goals (SDGs) for climate action and sustainable cities. ${ }^{7}$ Cities can also contribute to the decrease of the global carbon footprint owing to the consolidating urban population. ${ }^{8}$ However, their role in decarbonization is partially obfuscated by high diversity in socioeconomic status and biogeochemical cycles.

Motivated by anti-global warming action, scientists struggle to establish carbon mitigation approaches that can be applied to cities in different stages of development and with different economic structures, demographics, and climatic conditions. ${ }^{9-11}$ One increasingly important approach is to track both in-boundary and trans-boundary carbon emission associated with urban metabolic activities. ${ }^{12-16}$ However, this requires that the data needed for trade models (such as input-output tables) are accessible for cities. An alternative method is to consider carbon emissions embodied in products by fusing material flow analysis (MFA) and life-cycle analysis (LCA). ${ }^{17,18}$ In these methods, carbon emissions are quantified based on the energy and materials consumed by urban economic sectors. An important feature of this integrated approach is that it can be directly linked to carbon cycle models ${ }^{19-21}$ by placing emissions in a broader urban carbon metabolism. It is essential to track all physical carbon flows in cities because from a systemic perspective, all the activities in economic sectors including the consumption of carbon products (fossil fuels or non-fossil fuels products) will have an impact on carbon waste and emission via natural and economic transactions. ${ }^{21,22}$ In addition to existing 
51 carbon inventories, network-oriented model ${ }^{23,24}$ can provide a supplementary approach for 52 identifying carbon metabolic patterns in cities. ${ }^{25}$

Ecological network analysis (ENA) has been noted for its usefulness in uncovering flow structures and patterns in biological systems $\mathrm{s}^{26-28}$ and more recently, its adaptability in humandominated systems. ${ }^{23,24}$ ENA offers a set of powerful modelling approaches and metrics that have already been used to support decision making in sustainable resource management. ${ }^{29-31}$ There have been studies establishing ENA models to track carbon metabolic pathways associated ecological and economic activities in cities ${ }^{25,32,33}$ and applied them to show the possible pathways for more efficient spatial urban planning and carbon mitigation. ${ }^{34,35}$ Since ENA metrics do not directly represent the dynamics of economy, the effectiveness and implications of network metrics for the decarbonization of cities with highly diverse geographical and economic traits may be better understood on a comparative basis. ${ }^{36}$ In ecosystems, there frequently exists a common rule or pattern governing the carbon balance. ${ }^{37,38}$ It is natural to ask whether there are some common properties of carbon metabolic system comprising of natural and economic components and how they are linked to urban development and carbon emission mitigation. Currently, the interplay between metabolic properties and socioeconomic properties has only been assessed in one single city that are focused on carbon exchanges among economic sectors rather than all relevant urban components. ${ }^{39}$

This study establishes the carbon networks of eight global cities based on a city-level energy and material dataset, which captures carbon flows between urban economic sectors and natural components. Combing flow-based metrics and interpretative network metrics, we identify and compare the structure, patterns, and processes of urban carbon metabolism of high geographical and economic diversity. We further assess the relations of the system properties of carbon metabolic networks with socioeconomic attributes that are deemed important in urban development and planning. The two categories of metrics developed can be a promising scheme for linking carbon inventories to metabolic modelling efforts. Equipped with this networkoriented approach, we may be able to address how various carbon flows in cities can be 
concertedly managed according to urban economic and demographic changes.

\section{Materials and Methods}

\subsection{System diagram and technical framework}

A system diagram of the Carbon Flow Network (CFN) is shown in Figure 1. Urban carbon flows are embedded in an urban metabolic system wherein natural and artificial (humandominated) compartments are interacted with each other. These 13 aggregated compartments can be classified into four modules: 1) seven economic sectors, including Agriculture, forestry and horticulture (Agr), Mining (Min), Manufacturing (Man), Electricity, gas, and water (Ele), Construction (Con), Transportation (Tra), and Services (Ser); 2) two residential components, domestic consumption (Dom) and governmental consumption (Gov); 3) two components related to natural ecosystems: carbon stock variation (Sto) and biodegradable waste, such as food residues and other biodegradable waste (Dwa); 4) two components of environmental distribution: gaseous emissions (Ems) and non-biodegradable waste (Nwa). The CFN is established based on a quantification of inter-component carbon flows through the integration of material flow analysis (MFA), activity-based carbon inventory and life-cycle analysis (LCA).

4 We then assess the performance and pattern of a CFN and how they are related to the socioeconomic attributes based on two categories of metrics (flow-based metrics and interpretative network metrics). 


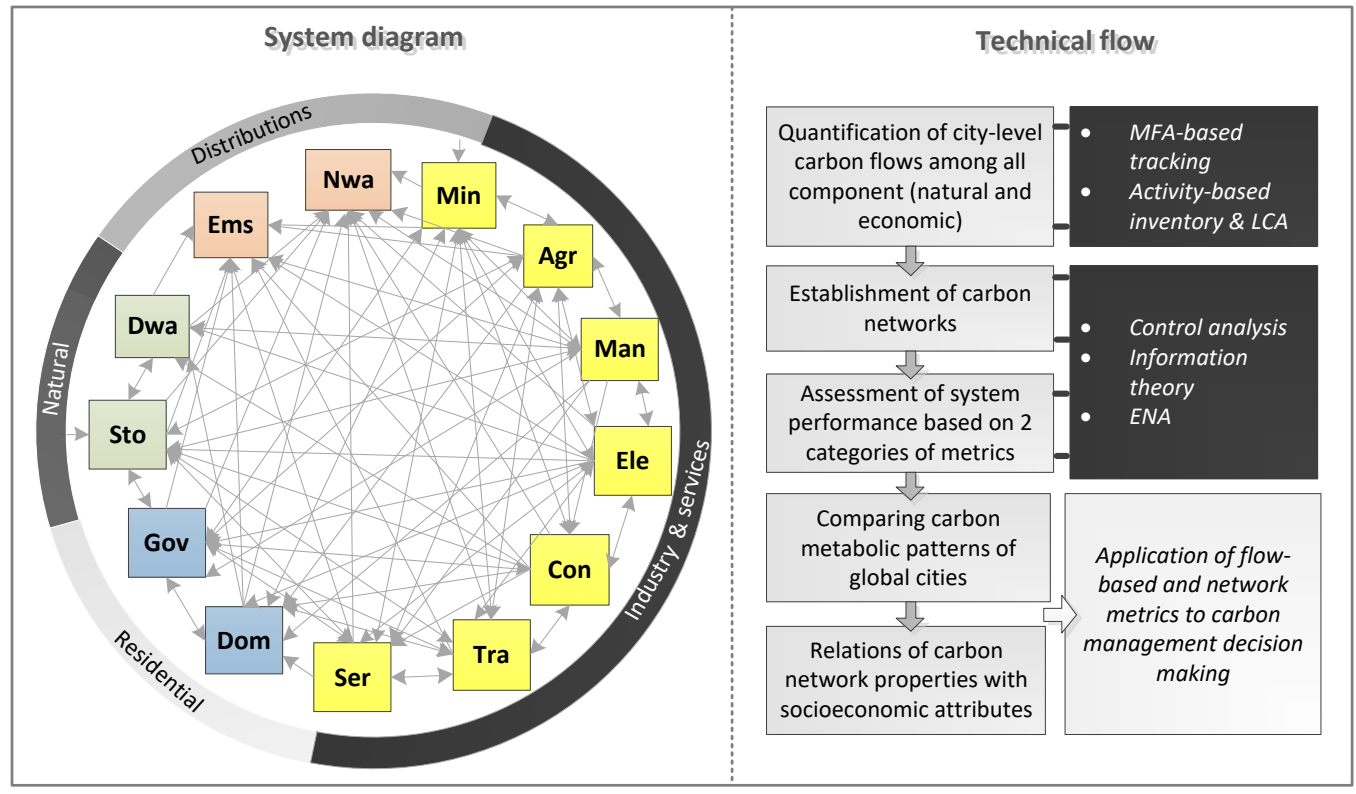

Figure 1 System diagram and technical framework for carbon flow network (CFN).

Note: Agr: Agriculture, forestry and horticulture; Min: Mining; Man: Manufacture; Ele: Electricity, gas and water; Con: Construction; Tra: Transportation; Ser: Services; Dom: Domestic consumption; Gov: Governmental consumption; Ems: gaseous emission; Dwa: biodegradable waste; Nwa: Nonbiodegradable waste; Sto: Stock variation. MFA: material flow analysis; LCA: life-cycle analysis; ENA: ecological network analysis

\subsection{Carbon flow inventory}

We used material flow analysis (MFA) to quantify the carbon flows and stock changes of urban economic sectors. MFA plays a significant role in determining the growth of urban metabolism, and can provide a strong foundation for assessing the impact of economic activities on natural ecosystems. ${ }^{18,40}$ Additionally, MFA has great potential for linking with global biogeochemical cycles ${ }^{41}$ In the carbon metabolic system of a city, it is important to consider the physical fluxes embedded in products and gaseous emissions from economic sectors (including in-boundary energy-use related emission as well as those emitted from the generation of imported electricity). These two parts form the major structure of a city's carbon profile, and are both considered in this study.

First, because direct carbon flow data are rare for most cities, we converted the mass-based 
115 flows in products (derived from local survey and published literature, see data compilation in

116 Section 2.6) to carbon flows by multiplying the material mass with a ratio called carbon content

117 factor (a) to obtain information on how much carbon is contained in assorted products. The 118 sector-specific carbon appropriation can be calculated from the aggregation of the product119 specific carbon contents, as follows:

$$
C_{i}=\sum_{x=1}^{n} \alpha^{x} M_{i}^{x}
$$

where $C_{i}$ is the carbon appropriated by an urban component; $M_{i}^{x}$ is the weight of a certain type of product $x$ consumed by component $i ; \alpha^{x}$ is the corresponding carbon content factor of that product. The carbon content factor varies in different types of products, such as fuel and biomass, agricultural and food products, and industrial and construction materials, as reported in the literature..$^{42-47}$ Forestry products used by the cities such as industrial roundwood and household wooden furniture are included in the carbon flow inventory, but the specific climatic impact of land use change is not considered.

Secondly, it is widely recognized that both in-boundary carbon emissions and crossboundary emissions from electricity consumption should be considered in urban carbon accounting. ${ }^{10,18}$ To calculate the flows of all urban components to Ems, we compiled an inventory of direct $\mathrm{CO}_{2}$ emissions from all economic sectors based on the approach recommended by the Intergovernmental Panel on Climate Change. ${ }^{48}$ The electricity-related carbon emissions outside of the urban boundary are also quantified and combined based on the respective carbon coefficients. The carbon flow to Ems is formulated as follows:

$$
C_{E m s(i)}=\sum_{k=1} E_{i}^{k} \times \omega_{i}^{k}+U_{i} \times \omega_{i}^{e l e}
$$

where $C_{E m s(i)}$ is the total amount of carbon emissions from economic sector $i$, and $E_{i}^{k}$ is the energy combustion from a certain fuel type or the intensity of a certain industrial process $(k)$; $\omega_{i}^{k}$ denotes the respective $\mathrm{CO}_{2}$ emissions coefficient for urban energy use or industrial 
processes; $U_{i}$ is the import of external electricity; $\omega_{i}^{\text {ele }}$ denotes the $\mathrm{CO}_{2}$ emissions coefficient

140 of electricity (depending on the energy mix in power generation).

\section{1}

\subsection{Establishment of carbon networks}

Fath and his colleagues ${ }^{49}$ proposed a step-by-step procedure for establishing ecological networks. This procedure includes three key processes: 1) determining the nodes and allowing to capture the interactions amongst different compartments; 2) quantifying the input, output, and throughflows between different compartments; 3) finalizing the network using a flowbalancing technique that has been widely applied to different types of networks. ${ }^{30}$ This can be extended to the development of urban carbon flow networks. Here, a node of the carbon network refers to the economic and ecological components of a city, while the arrow refers to the carbon flows between components. The carbon imported to one component is equal to the carbon transferred to other components through the production and consumption of products; that is, the sum of all carbon inflows is equal to the sum of all carbon outflows (the stock change is included as an outflow because it is considered as a component). In matrix terms, the row sum and column sum are the same. The system balance of the carbon flow network is expressed as follows:

$$
\begin{aligned}
& T_{i}^{\text {in }} \equiv z_{i}+\sum_{j=1} f_{j i} \\
& T_{i}^{\text {out }} \equiv \sum_{j=1} f_{i j}+y_{i} \\
& T_{i}^{\text {in }}=T_{i}^{\text {out }}
\end{aligned}
$$

where $T_{i}^{\text {in }}$ and $T_{i}^{\text {out }}$ represent the total amount of flow input to and output from each urban component, respectively; $f_{i j}$ is the carbon flow from component $i$ to $j ; z_{i}$ denotes the boundary inflows (external import) to component $i ; y_{j}$ denotes the boundary outflows (export to other regions) from component $j$. 
We adapted a set of system indicators from ecological network analysis (ENA) ${ }^{50}$ and information theory ${ }^{51}$ to identify the metabolic pattern of carbon flows and comprehensively assess the properties and functioning of carbon networks of cities. The application of networkbased indicators and tools in natural and human-dominated systems has been extensively discussed. ${ }^{39,52-55}$ In this study, we employed two categories of metrics to uncover the system properties of carbon flow networks and how they are related to the socioeconomic development in cities.

(1) Flow-based metrics (FBMs)

FBMs are represented by total system throughflow, boundary flow, cycled flow and Finn cycling index, which are grounded on physical laws and are widely used in the description of natural and human human-dominated systems.

The total system throughflow (TST) accounts for the sum of throughflows of all components. We used the TST of carbon to represent the size of a city's carbon metabolism, which does not only include gaseous emissions but also other physical carbon flows. Thus, we acquired a wider perspective with regard to how much carbon is appropriated by a city. The boundary flow (BF) is a subset of TST that captures the import of carbon from outside of the urban boundary, or the export of carbon to other regions or systems (in an equilibrium state these two are equal). This clarifies the reliance of urban carbon metabolism on external markets and ecosystems. The cycled flow (CF) can be derived from the diagonal elements of the integral flow matrix $(\mathrm{N})$, and is used to investigate the carbon cycled in the urban ecosystem through direct and indirect paths. The formulation of TST, BF, and CF is expressed as follows:

$$
\begin{aligned}
& \text { TST } \equiv \sum_{i=1} T_{i}^{\text {in }}=\sum_{i=1} T_{i}^{\text {out }} \\
& \text { BF }=\sum_{j=1} Z_{j}=\sum_{i=1} y_{i}
\end{aligned}
$$




$$
N=\left[n_{i j}\right]=\sum_{n=0}^{\infty} G^{n}=(\mathrm{I}-\mathrm{G})^{-1}
$$

$$
C F=\sum_{j=1}\left(\frac{n_{j j}-1}{n_{j j}} \mathrm{~T}_{j}\right)
$$

where $\mathrm{N}=\left[n_{i j}\right]$ is the integral dimensionless matrix of metabolic flow, and $\mathrm{G}$ is the direct dimensionless matrix of metabolic flow, ${ }^{50}$ where $g_{i j}=f_{i j} / T_{j}$

Finn cycling index $(\mathrm{FCI})^{56}$ was proposed to measure the amount of recycled flow compared with the total flow processed in a network, and was formulated based on the CF and TST results. Notably, FCI is not the recycling rate of carbon in the urban economy, but rather the carbon transferred amongst the components’ circular supply chains.

$$
F C I=\sum_{j=1}\left(\frac{n_{j j}-1}{n_{j j}} \mathrm{~T}_{j}\right) / \mathrm{TST}
$$

INMs include centrality, control allocation and dependence allocation, ascendancy, capacity, system robustness, synergism, which are based on theoretical ecological network models that need to be interpreted for applications in human-dominated systems. network component over another. ${ }^{26,59}$ Previous studies have demonstrated that NCA can effectively reveal inter-component relationships and dynamics, and identify the key processes in urban metabolic networks. ${ }^{34,60}$ This provides an advantage in targeting the most influential activities in terms of carbon emissions and waste, and can therefore assist in designing a more efficient method of urban decarbonization. In this study, we used the control metrics proposed by Chen and Chen, ${ }^{25}$ namely, the control allocation (CA) and dependence allocation (DA) to assess the control and dependence inter-component relationships with regard to urban carbon exchanges. 


$$
N^{\prime}=\left(\mathrm{n}_{i j}^{\prime}\right)=\sum_{n=0}^{\infty} G^{m}=\left(\mathrm{I}-\mathrm{G}^{\prime}\right)^{-1}
$$

$$
C A=\left[\mathrm{ca}_{i j}\right] \equiv\left\{\begin{array}{l}
n_{i j}-n_{j i}^{\prime}>0, c a_{i j}=\frac{n_{i j}-n_{j i}^{\prime}}{\sum_{i=1}^{m} n_{i j}-n_{j i}^{\prime}} \\
n_{i j}-n_{j i}^{\prime} \leq 0, c a_{i j}=0
\end{array}\right.
$$

$$
D A=\left[\mathrm{da}_{i j}\right] \equiv\left\{\begin{array}{l}
n_{i j}-n_{j i}^{\prime}>0, d a_{i j}=\frac{n_{i j}-n_{j i}^{\prime}}{\sum_{j=1}^{m} n_{i j}-n_{j i}^{\prime}} \\
n_{i j}-n_{j i}^{\prime} \leq 0, d a_{i j}=0
\end{array}\right.
$$

where $0 \leq d a_{i j}, c a_{i j} \leq 1 ; c a_{i j}$ indicates the control degree of compartment $j$ on compartment $i$ based on the controller's output environ; $d a_{i j}$ indicates the dependence degree of compartment $j$ on $i$ from the observer's input environ. In addition to $\mathrm{N}$, the output-oriented integral matrix $\mathrm{N}^{\prime}$ is also derived from a quantified CFN, wherein $G^{\prime}=\left(g_{i j}^{\prime}\right), g_{i j}^{\prime}=f_{i j} / T_{i}$. The control allocation (CA) and dependence allocation (DA) are determined by the two pairwise integral flows $\mathrm{N}$ and N'. In addition, the formulation of component importance represented by centrality ${ }^{61-64}$ is provided in Supporting Information.

The ascendancy (A) can quantify the network evolution and development built on the inter-component flows, and has been widely applied in assessing the organization, efficiency, and sophistication of various systems. ${ }^{31,57}$ Capacity (C) is often used to define the total volume of information that a network contains based on its size and self-organized flow pattern. On this basis, the relative ascendancy, or the ratio of ascendancy to capacity $(\alpha)$ has been proposed.$^{58}$ A higher A/C ratio value indicates a more developed, efficient, and organized system. ${ }^{24}$ In this study, the relative ascendancy represents the efficiency of carbon transfer and the transformation amongst different components.

$$
A=\operatorname{TST}_{P}^{2} \sum_{i, j}^{n} \frac{f_{i j}}{T S T_{P}} \log \frac{f_{i j} T S T_{P}}{T_{i} T_{j}}
$$




$$
C=-T S T_{P}^{2} \sum_{i, j}^{n} \frac{f_{i j}}{T S T_{P}} \log \frac{f_{i j}}{T S T_{P}}
$$

$$
\alpha=A / C
$$
where $\mathrm{U}$ is the integral utility matrix with consideration to both the direct and indirect relative

where $T S T_{P}$ is the total system throughput of a city's entire CFN, and the sum of all carbon imports, inter-component flows, and exports; A, C, and $\alpha$ denote the ascendancy, capacity, and relative ascendancy, respectively.

An ideal urban carbon network, arguably, should be both efficient in terms of the carbon exchanges amongst components and, at the same time, resilient against possible external disturbances (for example, lack of supply in certain carbon routes), which brings us to the fourth functional indicator called robustness (R). R measures the trade-off between efficiency and redundancy in a single metric. As the urban carbon networks move towards either extremes, i.e., overly efficient or overly redundant, the robustness of the carbon metabolic system falters.

$$
R=-\alpha \log (\alpha)
$$

Network synergism ${ }^{65}$ is an indicator extracted from utility analysis, and represents the ratio of the interaction effect between the benefit derived from net positive flows and the depression associated with net negative flows. In network utility analysis, the combination of element symbols in the integral utility matrix (U) can be used to determine the nature of interactions between two components, such as mutualism, competition, and so on. In this study, we focused on the ratio of positive conditions to negative conditions to obtain information on the health of and mutual benefit in an urban CFN.

$$
U=(\mathrm{I}-\mathrm{D})^{-1}
$$

$$
\text { flow difference; D is the direct utility matrix with consideration only to the direct relative flow }
$$


difference. The network synergism is calculated based on the ratio of the summing positive integral utilities to the summing negative integral utilities.

Based on these two categories of metrics, the correlations between system properties of carbon metabolism and urban socioeconomic attributes are assessed. A set of widely-used socioeconomic attributes that represent urban development are selected for correlation analysis, including carbon emission (in total or per capita), population, population density, GDP (Gross domestic products; in total or per capita), and urbanization rate. The significance of correlation may indicate the degree of relevance of the network metrics to current urban socioeconomic management and whether there is a synergy between carbon emission mitigation and urban metabolism optimization.

\subsection{Case study and data}

Eight global cities (at similar time point) were selected for case study: Vienna (2005), Sydney (2008), Sao Paulo (2009), Los Angeles (2008), London (2005), Hong Kong (2006), Cape Town (2006) and Beijing (2008). The geographical and socioeconomic situation of these eight cities are presented in Table S1. The selection of cities mainly because: (1) these cities cover all major populated regions (North America, South America, Europe, Asia, Oceania, and Africa), are currently in different development stages, and have sufficient geographical and socioeconomical diversity to test the generic pattern of urban carbon flows; (2) they have relatively reliable city-level energy and material data, which is a requirement for developing valid carbon flow models. A detailed data description for the urban CFNs is provided in Table S2, accompanied with the major sources of energy and material flow data for the eight cities.

\section{Results and discussion}

\subsection{Carbon flow networks of cities}

Figure 2 shows the inter-component flows in the carbon networks of the eight cities. The width of the ribbons indicates the amount of carbon exchanged between two urban components. 
277 The ribbons are colored according to the direction of the component from which the carbon is

278 exported. However, because the total output is equal to the total input in the balanced networks,

279 the number along each belt refers to the total carbon throughflow of each component. Note that 280 these flows refer to direct flows controlled by urban components, that is, a direct exchange of 281 carbon through trade or other linkages. We also show the per capita carbon throughflows of 8 global cities by component in Figure S1 in Supporting Information.

We find that import- and exported-related carbon flows contribute up to 70 percent of the cities’ total system throughflows. This indicates that urban carbon networks are highly open systems in the sense that they rely on the external environment through frequent imports of carbon as raw materials for manufacturing or household products for domestic consumption (and therefore export the carbon emissions to the atmosphere following use of the imports). The four dominant components inside the carbon networks of cities are Emission, Electricity, gas and water, Construction and Services, although the component contribution to the carbon flows is notably different for each city. Ems contributes the most to the total carbon throughflows in 291 most urban carbon networks (from 12\% in Sao Paulo to 19\% in Sydney). Two infrastructure292 related economic sectors, namely Electricity, gas and water and Construction, play an important 293 part in directing the carbon exchanges in the cities, and are responsible for $10 \%$ and $9 \%$ of the total carbon throughflow on average, respectively. The carbon emissions originating from power generation have been shown to be a significant source of urban carbon flows for cities in either developed or developing countries, although most flows originate outside of urban 297 boundaries. For example, in Vienna, flows from Electricity, Domestic consumption and 298 Transportation to Emission are the major carbon emission pathways. The carbon throughflow 299 of Construction is more diverse among cities. The construction activities in cities of developing 300 countries, such as Beijing and Sao Paulo, can contribute up to $12 \%$ of the total carbon 301 throughflow, while for cities in developed countries, such as Los Angeles and London, this proportion is only $8 \%$. This is mainly attributed to the higher demand of building materials (wood, cement, and so on) during fast urbanization in developing countries. Interestingly, Stock 
variation is a significant component for carbon networks in many cities, whose throughflow

305 accounting for $8 \%$ of the total carbon throughflow on average. In Sao Paulo, the amount of 306 carbon that ends up in stock (6229 kt C, 13\% of total system throughflow) is higher than that 307 becoming emission (5756 kt C). These components are associated with the biggest carbon flows 308 in the cities. In Sydney, flows from Electricity and Transportation to Emission and from 309 Construction to Stock variation are significant in the network, and the same occurs to Los 310 Angeles, London, and Cape Town. For Sao Paulo, the pairs of Construction $\rightarrow$ Stock variation 311 and Domestic consumption $\rightarrow$ Stock variation account for a large proportion of carbon 312 throughflows. The network analysis reveals important evidences proving that in addition to 313 gaseous emission, the change in urban stock may also have a significant impact on the whole 314 carbon networks of cities.

315 It is widely recognized that anthropogenic gaseous emissions play a major role in the 316 carbon cycles of natural-human complex systems such as cities. ${ }^{19,20}$ From an urban metabolism 317 perspective, our study demonstrated that approximately one-fifth of the total carbon 318 throughflow is directly associated with carbon emissions into the atmosphere. Additionally, the 319 significant flows to carbon stock raise concerns with regard to potential future emissions, 320 although they are not currently considered as accounting for part of the emissions. The 321 inventory of all inter-component carbon throughflows can offer a broader view of the size and 322 structure of urban carbon metabolism compared with carbon emissions accounting and provide 323 a basis for further carbon network modelling. 
Figure 2 Carbon flow networks of 8 global cities 
Notes: The number along each belt refers to the carbon throughflow (in kiloton of C) of each component

in the cities, while the percentages are their contributions to TST. Agr: Agriculture, forestry and horticulture; Min: Mining; Man: Manufacture; Ele: Electricity, gas and water; Con: Construction; Tra: Transportation; Ser: Services; Dom: Domestic consumption; Gov: Governmental consumption; Ems: gaseous emission; Dwa: Biodegradable waste; Nwa: Non-biodegradable waste; Sto: Stock variation; Row: rest of the world. The figure is powered by Circos Table Viewer.

\subsection{Performances and patterns at system and component levels}

Figure 3a shows the correlations between carbon flows and urban socioeconomics in total values. We found that three flow-based metrics, namely, the total system throughflow, boundary flow, and cycled flow, are highly correlated with the magnitudes of carbon emissions from cities. These flow metrics do not represent the carbon footprint of the urban economy (e.g. ${ }^{12,15}$ ); instead, they act as the carbon "metabolic intensity" and are affected by all carbon-related processes. Nonetheless, these flow-based metrics are closely related with carbon emissions in at least two ways: 1) the carbon emissions from various urban components are a significant part of total system throughflow, can contribute to the cycled flow when entering cycled chains, and subsequently become a fraction of the boundary flow; 2) more gaseous emissions often means higher consumption of energy or frequent industrial activity, which in turn attracts carbon inflow to a city as fuels, construction materials, and other products. More importantly, the deviation of these indicators from carbon emissions is meaningful. These metrics can provide useful information on a city's total metabolism, boundary metabolism, and cycled metabolism, which cannot be obtained by direct carbon accounting. The cycled flow is also a good measure for the degree of circularity in the economy, a concept that gaining traction as a way to both strengthen the economy and lower emissions.

The total system throughflow, boundary flow, and cycled flow have strong positively linear correlations with the population. This indicates that the impact of the urban population on carbon metabolism is unlikely to slow down as more people swarm into the city. An exception is Sao Paulo with a population of 11.4 million, whose total system throughflow, boundary flow, and cycled flow are lower than the values predicted by the regression model. Sao Paulo has a relatively low-carbon economy from a carbon metabolic flow perspective. In contrast, Sydney and Los Angeles have a 
higher level of carbon flow compared with the predicted level, which indicates a relatively highcarbon city profile given their carbon emissions related to transportation. Here, the correlations of GDP with the total system throughflow and boundary flow are much weaker. The expansion of the economic scale does not have a definitive impact on the urban carbon metabolism. Many other factors may also be equal or more important, such as technology, scale of export, and so on. Essentially, there is no significant correlation between GDP and cycled flow, in the sense that cycled chains are often more related to the economic structure and compactness of urban industries and services. This implies that, for cities with a larger amount of emission from transportation sector like Los Angeles, the carbon metabolism is more intensive than other study cities.

Figure 3b shows the correlation between the carbon metabolic function (Finn cycling index, synergism, system robustness) and the urban socioeconomics of the eight cities. The Finn cycling index for the eight cities ranges from 0.06 to 0.10 , which indicates that less than $10 \%$ of the carbon is cycled within the urban metabolic network. We found that Finn cycling index has a positive correlation with the population density. This suggests that it is possible for the recycling of carbon products in cities to increase with a denser urban form and more compact industrial network. In contrast, Finn cycling index has a negative correlation with GDP per capita and carbon emissions per capita, although this correlation is loose. Higher GDP may

371 result in larger total system throughflow, but the increase of financial income typically 372 augments gaseous emissions that are not cycled back into the urban economy. This is an 373 important side effect caused by urban economic development. Therefore, a goal to increase the 374 service economy, because on face it might have lower direct emissions, might lead to higher overall emissions both because GDP per capita increases and lower cycling. Similarly, the network synergism of cities is positively correlated with the population density, and negatively 377 correlated with the carbon emissions per capita. Cities with a higher population density and 378 lower per capita carbon emissions also have a healthier carbon metabolic system with better 379 cooperation amongst components. This demonstrates that the objectives of urban 380 decarbonization and carbon metabolism optimization can be simultaneously achieved in a 381 systemic urban carbon management framework. The cities’ relative ascendancy $(\alpha)$ ranges from 
0.22 to 0.25 , resulting in variations in the system robustness between cities (with up to $5 \%$ difference between Sydney and Vienna). The average system robustness of carbon networks is 0.34 of the study cities, which falls in the middle of natural ecosystems and artificial or economic trade systems (as shown by Figure S3 in Supporting Information). This is mainly because the carbon flow networks of cities represent the interface between natural processes (such as waste decomposition and carbon sequestration by urban trees) and socioeconomic activities (such as energy-related emission, carbon exchange in products and food consumption) and can be influenced by both natural and human-dominated components in cities. We find that network metrics used such as system robustness and synergy does not have a significant linear correlation with either per capita GDP, population density, or per capita carbon emission.

However, it is important to note that the complexity and evolution of urban metabolic networks are not fully determined by social and economic conditions. This indicates that the metabolic properties of carbon networks may not evolve in the same pace as urban socioeconomic development, and the interpretive network metrics usually used in biological or ecological systems, could not be interpreted in the same way as other mass-based network indicators. In particular, system robustness has a clear linkage to ecosystem function (e.g. biodiversity and abundance) and implications that may hard to find parallel in socioeconomic systems like urban economy. Thus, caution should be used when applying system robustness to assess urban carbon metabolism or possibly other urban systems that are driven by socioeconomic activities rather than ecological processes. 

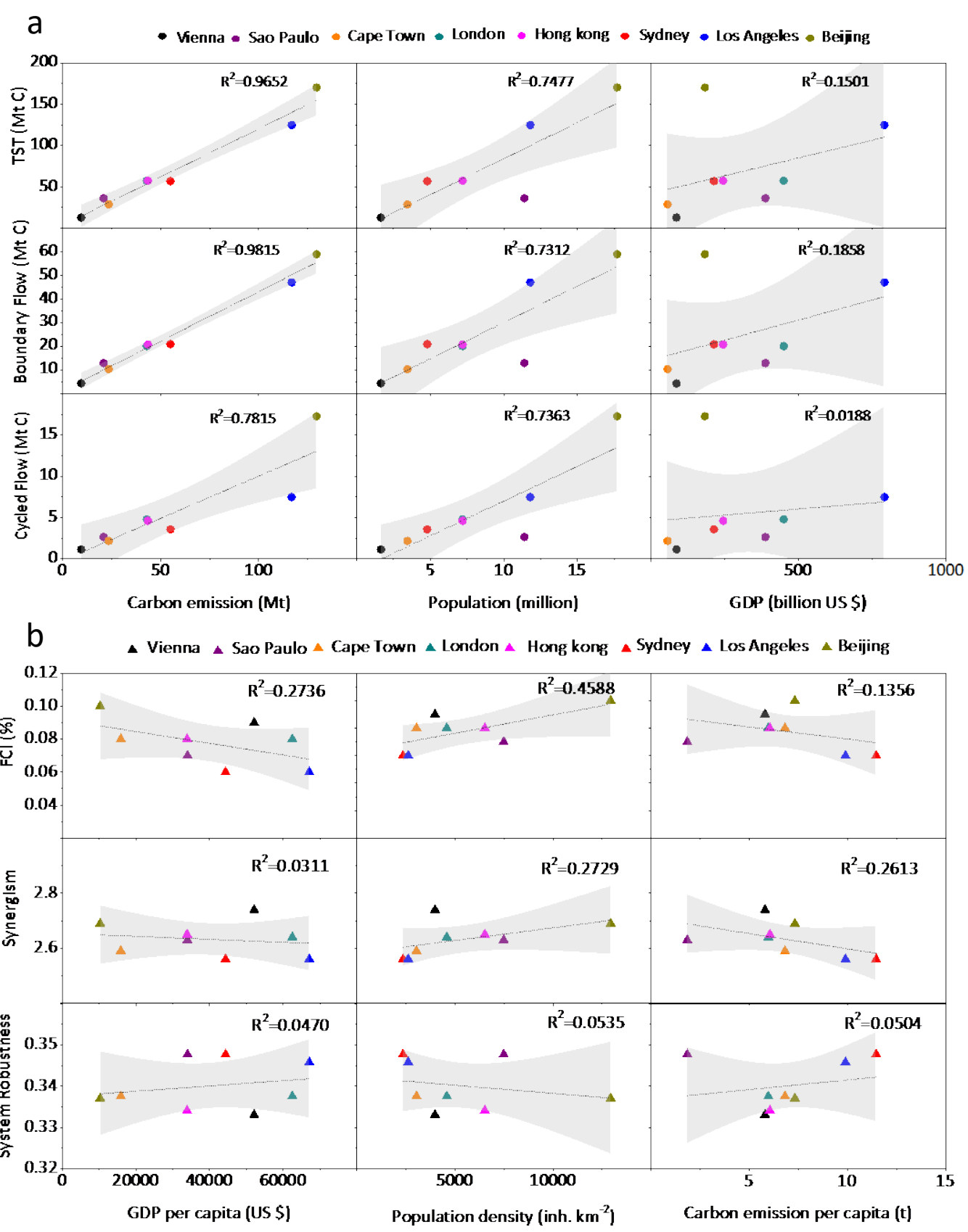

Figure 3 Correlations (a) between carbon metabolic flows and urban socioeconomics, and (b) between carbon metabolic function and urban socioeconomics. Extended regression results are provided in Table S3 and Table S4 in Supporting Information.

Based on a network metrics called centrality, we assessed the role each component plays for the eight cities with consideration to both direct and indirect flows (Figure S2). Generally, Agriculture, Mining, Transportation, and Biodegradable waste are more important from an input perspective, in the sense that their input environ centralities are notably higher than their 
output environ centralities. This phenomenon is common for all study cities and may indicate

413 the similarity of carbon metabolizing behaviors in specific components regardless of the city's

414 development stage. However, Emission component, Stock variation, and Non-biodegradable

415 waste are dominant ways of exporting carbon with relatively high output environ centralities.

416 The Emission component is more important in some cities such as Sydney, Los Angeles, and

417 London, while Stock variation seems to contribute more in Sao Paulo. This confirms our

418 conjecture that Gaseous emission and Urban stock are two significant destinations of the

419 integral carbon flow. Emission, Construction, and Stock variation have the largest throughflow

420 centrality in Vienna, Sydney, and Sao Paulo, while in Los Angeles, London, Hong Kong, and

421 Cape Town, Stock variation and Service are dominant in addition to Emission. A further

422 evaluation of these two components is needed to clarify how they are controlled by other

423 components and what is the most efficient approach toward regulating them.

The control and dependence relationships between components in urban carbon networks reveal the potential mechanism of efficient carbon management (Figure 4). By considering all direct and indirect interactions, we found that the control allocation (CA) amongst the components are diverse and uneven. The silence (low degree of control relations) amongst the economic sectors does not mean that there are no interactions, but rather that the interactions between the urban economy and the environmental distributions are more intensive from a network control perspective. There are significant differences between the control regimes of the eight global cities. However, various generic patterns can be derived. By targeting the dominant components and key processes in the carbon networks, it is possible to obtain efficient 433 carbon mitigation pathways in cities. Many urban economic sectors such as Electricity, gas and 434 water, Transportation, and Construction have a strong control over Emission and Stock 435 variation. In some cases, more control in these economic sectors is exerted over Emission. For example, in Sydney, 32\% of Electricity, gas and water control in carbon exchange is allocated 437 to Emission, which is much higher than that received by Stock variation. However, in Sao Paulo, more than $70 \%$ of the control in the economic sectors is allocated to Stock variation, owing to 
the dominance of stock in the carbon network (as revealed by previous results). The economic sectors also show some extent of control over Domestic consumption, while in most cities, Domestic consumption has significant control over Emission and Stock variation, but no control over the industry and Service. For example, 35\% and 51\% of Domestic consumption control goes to Emission and Stock variation in Vienna, respectively, while the proportions in Sydney are $26 \%$ and $45 \%$, respectively.

The dependence allocation (DA) shows the inter-component control relationships in the carbon networks from a receiver's viewpoint. Manufacturing, Service, and Domestic consumption depend on many other components in the urban economy to derive carbon. For example, the Manufacturing in Sao Paulo depends on Construction, Mining, and Service by $65 \%, 6 \%$, and $5 \%$, respectively. Service is greatly dependent on Electricity, gas and water and Construction, with a total dependence degree of 81-91\%. In London, Domestic consumption is dependent on Electricity, gas and water, Construction, Transportation, and Service by 13\%, $20 \%, 11 \%$, and $44 \%$, respectively. However, this varies from city to city. For example, Hong Kong's Domestic consumption is dependent on these components by 6\%, 35\%, $12 \%$, and 31\%, respectively. In London, the dependence of domestic activities on Service is up to $44 \%$ owing to the major role of the commercial activities in the city’s economy. We can see that Emission depend on a range of urban economic sectors, namely Electricity, gas and water, Construction, Transportation, and Service. In our sample of cities, the Emission is controlled by these economic sectors by 16-22\%, 7-12\%, 9-16\%, 8-15\%, and 6-18\%, respectively. Additionally, 459 domestic activities also have a considerable impact on Emission with a dependence degree of 460 6-10\% amongst the cities. We also found that Stock variation is very dependent on 461 Construction, Service, and Domestic consumption in the sense that these three components are amongst the major sources of carbon stored in the urban economy. It is essential to have a clear understanding of the full carbon flow chains before they end up in emissions, including both direct and indirect pathway between sectors. ${ }^{25,34}$ These results can provide a systemic perspective on how carbon emissions are controlled by urban economic sectors through 


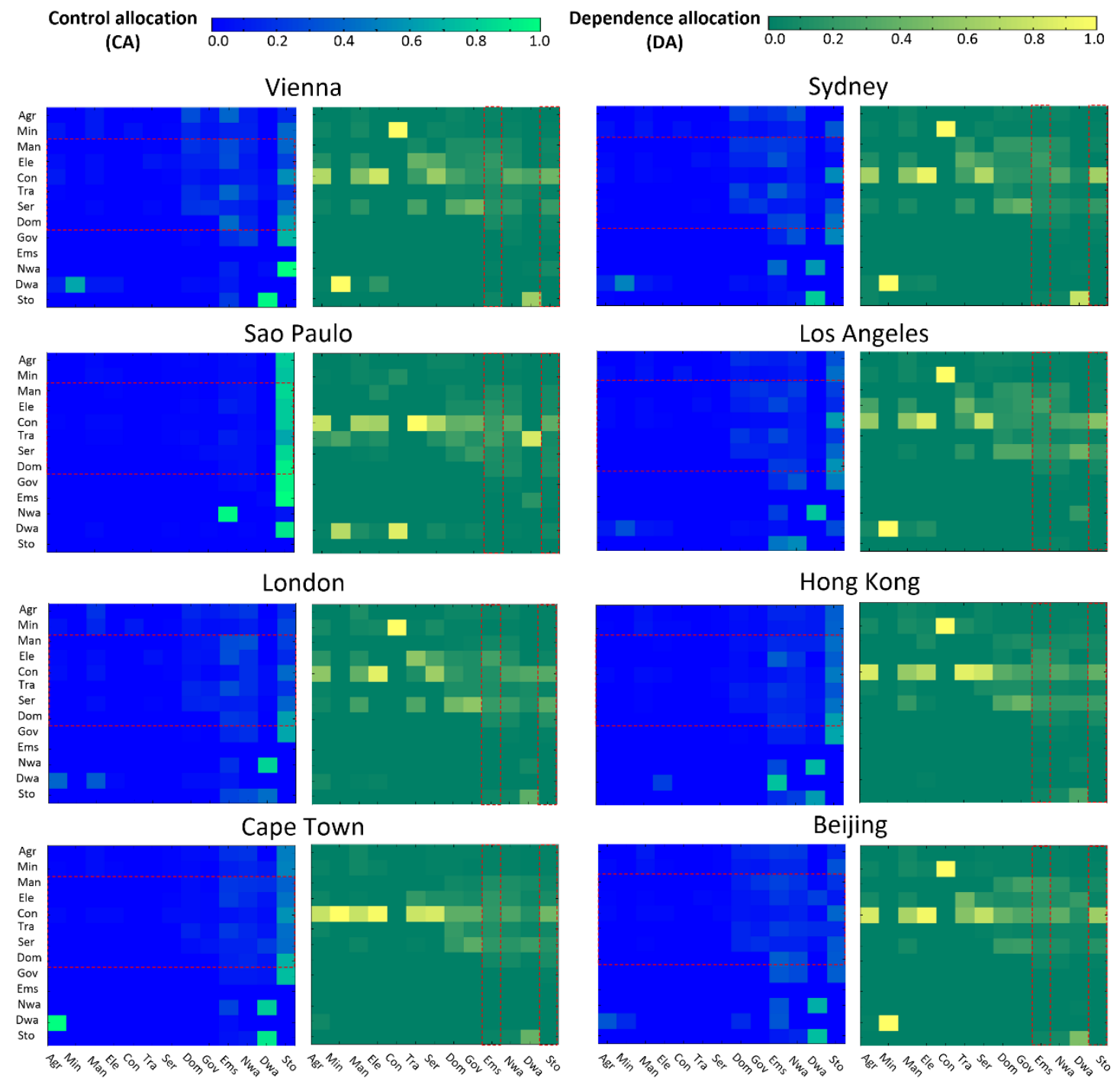

468 Figure 4 Control allocation (CA) and dependence allocation (DA) between urban components 469 in exchanging carbon flows. Note: CA should be read from row to column, i.e. the control of 470 column components over the row components; DA should also be read from row to 471 column, but the meanings change to the dependence of column components on the row 472 components. Both CA and DA are within the range between 0 and 1, with larger number 473 representing higher control or dependence over others. The highlighted areas are the 474 control of key urban economic sectors over others from the CA perspective, and the 475 dependence of carbon emission and stock on other sectors from the DA perspective.

476 Cities must address climate change in every possible way. ${ }^{36}$ Yet, there is a big knowledge 
gap between the inventory of carbon emissions/sinks and the modelling of carbon flows within

478 the context of urban metabolism. A number of methodological frameworks and guidelines have 479 been proposed for city-level carbon emission inventory. ${ }^{12,18,66-68}$ The analyses centered around emission dominates current discussion on urban decarbonization, while other non-emission carbon being exchanged in urban economy are largely disregarded. This is mainly because how these flows will end up in emission is not clearly understood and there is no sufficient and reliable data to do just so.

On the other hand, there has been increasing interest in using a nature-based method to alleviate the environmental burden carried by urban development. ${ }^{69,70}$ The concept of metabolism fits right into this research initiative. Urban metabolism has been developed as a methodological framework for investigating various energy and material flows associated with urban growth. ${ }^{71}$ The human impact on urban carbon metabolism is highly complex and interrelated with various natural and economic components. To better understand this impact, all carbon imports, exports and inter-component exchanges comprising the network should be examined. Current inventory-based approaches are mainly directed to an intensity-based analysis, and yet the structural and functional aspects can be better understood by modelling techniques that include indirect effects. The integration of intensity, structural and functional information of carbon metabolism is needed to fill in current knowledge gap and provide a broader understanding of cities’ impact on climate change. . $^{10,18,33,72}$

The categorization of carbon flow metrics in this study (flow-based metrics and interpretative network metrics) can provide a promising scheme for linking carbon emission inventories to metabolic modelling efforts. Flow-based metrics are grounded on conservation 499 of mass and so these results can be directly used in making carbon management policies, which 500 are not very different from indicators like carbon emissions and sinks for this matter. In contrast, 501 interpretative network metrics can be used to understand the mechanism of network functioning 502 or inter-component relations that cannot be shown by flow-based metrics. They are appropriate 503 for interpreting the carbon-related interactions in the urban metabolic system and for comparing 
the system performances of carbon metabolic networks among cities, though whether they can

505 be employed in the regulation of sectoral activities and behaviors needs further inspection. This

506 merit is well-reflected by the control analysis it provides. Regarding carbon analyses, one can

507 account for direct carbon emissions from all economic sectors and households using material

508 flow analysis and embodied carbon emission from input-output analysis, but how the carbon

509 emission of a sector is controlled by activities of a bunch of other sectors and how these

510 activities are further induced by other activities could be only be fully addressed using network

511 analysis. Another merit is that it can provide potential goal functions for system evolution and

512 optimization such as maximum ascendency, maximum cycling, among others. ${ }^{73}$ Some of these

513 goal functions are showing potential of applications in socioeconomic systems as well, such as

514 the information-based carbon modelling in. ${ }^{74}$ Our results indicate that they align favorably with

515 other common standard metrics already in place, but give a richer insight into how the network

516 patterns lead to these outcomes. Within the framework of urban metabolism, these two

517 categories of metrics can be combined to quantify the influence of urbanization and economic

518 transition on carbon network connectivity and diversity, ${ }^{31,74}$ and foster system-oriented

519 strategies for urban carbon reduction that supplement current mitigation actions.

520 Globally, comparative studies among cities are called for to disentangle the interactions

521 of human activities and to find strategies and roadmap to mitigate climate change. ${ }^{36,75,76}$ The

522 analyses of eight global cities suggest there is a large difference in city-level carbon balance,

523 but a similarity in inter-component relationships and general metabolic characteristics can be

524 found. An integration of flow-based metrics, interpretative network metrics and other

525 socioeconomic models will convey important information about how future carbon flows

526 should be managed according to the urban economic and demographic changes. A major

527 limitation to the comparative results is the relatively small sample used (eight cities). Provided

528 the metabolic data are more available and frequently updated at a city level, a global

529 comparation with a large sample may renew our current understanding. Still, the current eight-

530 city study is able to demonstrate how the carbon metabolic patterns can be identified and 
531 compared among different cities. Policy makers can acquire the carbon metabolism knowledge

532 from other cities to help them select their own strategies and countermeasures and guide cities

533 toward more rational and concerted climate actions. In turn, this will increase the importance

534 of determining the key metabolic characteristics of different cities and using them as a reference

535 during the adaptation of available mitigation techniques.

\section{Acknowledgements}

This work was supported by the Natural Science Funds for Distinguished Young Scholar of Guangdong Province, China (2018B030306032), National Natural Science Foundation of China (71704015), the National Science Fund for Distinguished Young Scholars of China (71725005), the National Key Research \& Development Program (2016YFA0602304) and the Fundamental Research Funds for the Central Universities. We appreciate the comments from Prof. Klaus Hubacek, Dr. Kuishuang Feng and Prof. Laixiang Sun on the early idea of this paper.

\section{References}

[1] Grimm, N. B.; Faeth, S. H.; Golubiewski, N. E.; et al. Global change and the ecology of cities. Science 2008, 319(5864), 756-760.

[2] Gamba, P.; Herold, M. Global mapping of human settlement: experiences, datasets and prospect; CRC Press, Boca Raton, 2009.

[3] Seto, K. C.; Dhakal, S.; Bigio, A.; et al. Human Settlements, Infrastructure and Spatial Planning. In: Climate Change 2014: Mitigation of Climate Change. Contribution of Working Group III to the Fifth Assessment Report of the Intergovernmental Panel on Climate Change [Edenhofer, O., R. et al. (eds.)]. Cambridge University Press, Cambridge, United Kingdom and New York, NY, USA, 2014.

[4] Seto, K. C.; Güneralp, B.; Hutyra, L. R. Global forecasts of urban expansion to 2030 and direct impacts on biodiversity and carbon pools. Proceedings of the National Academy of Sciences 2012, 109, 16083-16088.

[5] American Association for the Advancement of Science, Rise of the city. Science 2016, 352(6288), 906-907.

[6] United Nations Climate Change. The Paris Agreement. Available at: https://unfccc.int/process- 
and-meetings/the-paris-agreement/the-paris-agreement.

[7] UN-Habitat; UNESCO; World Health Organization; UNISDR; UN Women; UNEP and UNDP. SDG Goal 11 Monitoring Framework. Accessed at http://unhabitat.org/sdg-goal-11monitoring-framework/; 2016.

[8] Wigginton, N. S.; Fahrenkamp-Uppenbrink, J.; Wible, B.; et al. Cities are the future. Science 2016, 352 (6288), 904-905.

[9] Reckien, D.; Creutzig, F.; Fernandez, B.; et al. Climate change, equity and the Sustainable Development Goals: an urban perspective. Environment and urbanization 2017, 29(1), 159182.

[10] Creutzig, F.; Baiocchi, G.; Bierkandt, R.; et al. Global typology of urban energy use and potentials for an urbanization mitigation wedge. Proceedings of the National Academy of Sciences 2015, 112 (20), 6283-6288.

[11] Chen, S. Q.; Chen, B. Coupling of carbon and energy flows in cities: A meta-analysis and nexus modelling. Applied energy 2017, 194, 774-783.

[12] Chavez, A.; Ramaswami, A. Articulating a trans-boundary infrastructure supply chain greenhouse gas emission footprint for cities: Mathematical relationships and policy relevance. Energy Policy 2013, 54, 76-384.

[13] Ramaswami, A.; Tong, K.; Fang, A.; et al. Urban cross-sector actions for carbon mitigation with local health co-benefits in China. Nature Climate Change 2017, 7(10), 736.

[14] Liu, Z.; Feng, K.; Hubacek, K.; et al. Four system boundaries for carbon accounts. Ecological modelling 2015, 318, 118-125.

[15] Chen, S. Q.; Liu, Z.; Chen, B.; et al. Dynamic Carbon Emission Linkages Across Boundaries. Earth's Future 2019,7(2), 197-209.

[16] Lin, J.; Hu, Y.; Cui, S.; et al. Tracking urban carbon footprints from production and consumption perspectives. Environmental Research Letters 2015, 10(5), 054001.

[17] Ramaswami, A.; Hillman, T.; Janson, B.; et al. A Demand-Centered, Hybrid Life-Cycle Methodology for City-Scale Greenhouse Gas Inventories. Environmental Science \& Technology 2008, 42(17), 6455-6461.

[18] Kennedy, C.; Steinberger, J.; Gasson, B.; et al, Greenhouse gas emissions from global cities. Environmental Science \& Technology 2009, 43, 7297-7302.

[19] Pataki, D. E.; Alig, R. J.; Fung, A. S.; et al. Urban ecosystems and the North American carbon cycle. Global Change Biology 2006, 12, 2092-2102.

[20] Churkina, G.; Brown, D. G.; Keoleian, G. Carbon stored in human settlements: the conterminous United States. Global Change Biology 2010, 16, 135-143.

[21] Churkina, G. Modeling the carbon cycle of urban systems. Ecological Modelling 2008, 216, 107-113.

[22] Peters, G. P.; Davis, S. J.; Andrew, R. A synthesis of carbon in international trade. 
Biogeosciences 2012, 9, 3247-3276.

[23] Schramski, J. R.; Dell, A. I.; Grady, J. M.; et al. Metabolic theory predicts whole-ecosystem properties. Proceedings of the National Academy of Sciences 2015, 112(8), 2617-2622.

[24] Pizzol, M.; Scotti, M.; Thomsen, M. Network Analysis as a tool for assessing environmental sustainability: Applying the ecosystem perspective to a Danish Water Management System. Journal of environmental management 2013, 118, 21-31.

[25] Chen, S.; Chen, B. Network environ perspective for urban metabolism and carbon emissions: A case study of Vienna, Austria. Environmental Science \& Technology 2012, 46(8), 4498-4506.

[26] Schramski, J. R.; Gattie, D. K.; Patten, B. C.; et al. Indirect effects and distributed control in ecosystems: Distributed control in the environ networks of a seven-compartment model of nitrogen flow in the Neuse River Estuary, USA—Steady-state analysis. Ecological Modelling 2006, 194(1), 189-201.

[27] Hines, D. E.; Singh, P.; Borrett, S. R. Evaluating control of nutrient flow in an estuarine nitrogen cycle through comparative network analysis. Ecological Engineering 2016, 89, 70-79.

[28] Rakshit, N.; Banerjee, A.; Mukherjee, J.; et al. Comparative study of food webs from two different time periods of Hooghly Matla estuarine system, India through network analysis. Ecological Modelling 2017, 356, 25-37.

[29] Layton, A.; Bras, B.; Weissburg, M. Designing industrial networks using ecological food web metrics. Environmental science \& technology 2016, 50(20), 11243-11252.

[30] Borrett, S. R; Sheble, L.; Moody, J.; et al. Bibliometric review of ecological network analysis: 2010-2016. Ecological Modelling 2018, 382, 63-82.

[31] Fath, B. D. Quantifying economic and ecological sustainability. Ocean \& Coastal Management 2015, 108, 13-19.

[32] Lu, Y.; Chen, B.; Feng, K.; et al. Ecological network analysis for carbon metabolism of ecoindustrial parks: a case study of a typical eco-industrial park in Beijing. Environmental Science \& Technology 2015, 49(12), 7254-7264.

[33] Xia, C.; Li, Y.; Xu, T.; et al. Quantifying the spatial patterns of urban carbon metabolism: A case study of Hangzhou, China. Ecological Indicators 2018, 95, 474-484.

[34] Chen, S. Q.; Chen, B. Tracking inter-regional carbon flows: a hybrid network model. Environmental Science \& Technology 2016, 50 (9), 4731-4741.

[35] Xia, L.; Zhang, Y.; Wu, Q.; et al. Analysis of the ecological relationships of urban carbon metabolism based on the eight nodes spatial network model. Journal of Cleaner Production 2017, 140, 1644-1651.

[36] Bai, X.; Dawson, R. J.; Ürge-Vorsatz, D.; et al. Six research priorities for cities and climate change. Nature 2018, 555, 23-25.

[37] Falkowski, P.; Scholes, R. J.; Boyle, E.; et al. The global carbon cycle: a test of our knowledge of earth as a system. Science 2000, 290(5490), 291-296. 
[38] Luo, Y.; Keenan, T. F.; Smith, M. Predictability of the terrestrial carbon cycle. Global change biology 2015, 21(5), 1737-1751.

[39] Chen, S.; Xu, B.; Chen, B. Unfolding the interplay between carbon flows and socioeconomic development in a city: what can network analysis offer? Applied Energy 2018, 211, 403-412.

[40] Piña, W. H. A.; Martínez, C. I. P. Urban material flow analysis: An approach for Bogotá, Colombia. Ecol Indicat 2013. http://dx.doi.org/10.1016/j.ecolind.2013.10.035

[41] Bai, X. Industrial ecology and the global impacts of cities. Journal of Industrial Ecology 2007, 11(2), 1-6.

[42] Hao, Y.; Su, M.; Zhang, L.; et al. Integrated accounting of urban carbon cycle in Guangyuan, a mountainous city of China: the impacts of earthquake and reconstruction. Journal of Cleaner Production 2015, 103, 231-240.

[43] DAFF. Australian State of the Forests Report: Five yearly report 2008. Australian Bureau of Agricultural and Resource Economics and Sciences. Department of Agriculture, Forestry and Fisheries, 2008.

[44] Lamlom, S. H.; Savidge, R. A. A reassessment of carbon content in wood: variation within and between 41 North American species. Biomass and Bioenergy 2003, 25, 381-388.

[45] Stockmann, K. D.; Anderson, N. M.; Skog, K. E.; et al. Estimates of carbon stored in harvested wood products from the United States forest service northern region, 1906-2010. Carbon Balance and Management 2012, 7, 1.

[46] Moriarty, D. J.; Barclay, M. C. Carbon and Nitrogen Content of Food and the Assimilation Efficiencies of Penaeid Prawns in the Gulf of Carpentaria. Aust. J. Mar. Freshwater Res 1981, 32, 245-251.

[47] Aguilera, J. A.; Aragon, C.; Campos, J. Determination of carbon content in steel using laserinduced breakdown spectroscopy. Applied Spectroscopy 1992, 46, 1382-1387.

[48] IPCC, Revised 1996 Guidelines for National Greenhouse Gas Inventories: Workbook (1997). http://www.ipcc-nggip.iges.or.jp/public/gl/invs1.html, 1997.

[49] Fath, B. D.; Scharler, U. M.; Ulanowicz, R. E.; et al. Ecological network analysis: network construction. Ecological Modelling 2007, 208(1), 49-55.

[50] Fath, B. D.; Patten, B. C. Review of the foundations of network environ analysis. Ecosystems 1999, 2(2), 167-179.

[51] Ulanowicz, R. E. Mass and energy flow in closed ecosystems. Journal of Theoretical Biology 1972, 34(2), 239-253.

[52] Fath, B. D.; Borrett, S. R. A Matlab ${ }^{\circledR}$ function for network environ analysis. Environmental Modelling \& Software 2006, 21(3), 375-405.

[53] Kazanci, C. EcoNet, a new software for ecological model simulation and network analysis. Ecological Modelling 2007, 208 (1), 3-8.

[54] Schramski, J. R.; Kazanci, C.; Tollner, E. W. Network environ theory, simulation, and EcoNet ${ }^{\circledR}$ 
2.0. Environmental Modelling \& Software 2011, 26(4), 419-428.

[55] Borrett, S. R.; Lau, M. K. enaR: an R package for ecosystem network analysis. Methods in Ecology and Evolution 2014, 5(11), 1206-1213.

[56] Finn, J. T. Measures of ecosystem structure and function derived from analysis of flows. $J$. Theor. Biol. 1976, 56, 363-80.

[57] Ulanowicz, R. E.; Norden, J. S. Symmetrical overhead in flow networks. Int J Syst Sci 1990, 21(2), 429-37.

[58] Ulanowicz, R. E.; Goerner, S. J.; Lietaer, B.; et al. Quantifying sustainability: resilience, efficiency and the return of information theory. Ecological Complexity 2009, 6 (1), 27-36.

[59] Fath, B. D. Distributed control in ecological networks. Ecol Model 2004, 179, 235-46.

[60] Chen, S. Q.; Fath, B. D.; Chen, B. Information-based Network Environ Analysis: A system perspective for ecological risk assessment. Ecol Indic 2011, 11 (6), 1664-1672.

[61] Borgatti, S. P. Centrality and network flow. Soc Networks 2005, 27, 55-71.

[62] Wasserman, S.; Faust, K. Social Network Analysis: Methods and Applications. Cambridge University Press, Cambridge, New York, 1994.

[63] Borrett, S. R. Throughflow centrality is a global indicator of the functional importance of species in ecosystems. Ecological Indicators 2013, 32, 182-196.

[64] Fann, S. L.; Borrett, S. R. Environ centrality reveals the tendency of indirect effects to homogenize the functional importance of species in ecosystems. (Report). Journal of Theoretical Biology 2012, 294, 74-86.

[65] Fath, B. D.; Patten, B. C. Network synergism: emergence of positive relations in ecological systems. Ecological Modelling 1998, 107(2-3), 127-143.

[66] Kennedy, A.; Ibrahim, N.; Hoornweg, D. Low-carbon infrastructure strategies for cities. Nature Climate Change 2014, 4, 343-346.

[67] WRI and WBCSD. The Greenhouse Gas Protocol: A Corporate Accounting and Reporting Standard, World Resources Institute and World Business Council for Sustainable Development: Washington, DC. http://www.ghgprotocol.org/sites/default/files/ghgp/standards/ghg-protocolrevised.pdf, 2004.

[68] ICLEI, WRI and C40. Global Protocol for Community-Scale GHG Emissions. http://www.iclei.org/activities/agendas/low-carbon-city/gpc.html, 2014.

[69] Collins, J. P.; Kinzig, A.; Grimm, N. B.; et al. A New Urban Ecology Modeling human communities as integral parts of ecosystems poses special problems for the development and testing of ecological theory. American Scientist 2000, 88(5), 416-425.

[70] Kabisch, N.; Frantzeskaki, N.; Pauleit, S.; et al. Nature-based solutions to climate change mitigation and adaptation in urban areas: perspectives on indicators, knowledge gaps, barriers, and opportunities for action. Ecology and Society 2016, 21(2), 39.

[71] Kennedy, C.; Cuddihy, J.; Engel-Yan, J. The changing metabolism of cities. Journal of 
Industrial Ecology 2007, 11(2), 43-59.

[72] Chen, S.; Chen, B.; Feng, K.; et al. Physical and virtual carbon metabolism of global cities. Nature Communications 2020, 11, 182.

[73] Fath, B. D.; Patten, B. C.; Choi, J. S. Complementarity of ecological goal functions. Journal of theoretical biology 2001 208(4), 493-506.

[74] Fang, D.; Chen, B. Information-based ecological network analysis for carbon emissions. Applied Energy 2019, 238, 45-53.

[75] Bai, X. Eight energy and material flow characteristics of urban ecosystems. Ambio. 2016, 45(7), 819-830.

[76] C40 Cities Climate Leadership Group. https://www.c40.org/. 